\title{
Transforming the AEC Industry: A Model-Centric Approach
}

\author{
Hala Nassereddine ${ }^{1}$, Mahmoud El Jazzar ${ }^{2}$ and Melanie Piskernik ${ }^{3}$
}

1 University of Kentucky, Lexington, USA, hala.nassereddine@uky.edu

2 University of Kentucky, Lexington, USA, meljazzar@uky.edu

3 Technical University of Vienna, Vienna, Austria, melanie.piskernik@tuwien.ac.at

\begin{abstract}
The increased complexity of construction projects requires the involvement of a wide range of organizations throughout the project lifecycle - from conceptual planning to decommissioning. Construction project teams, therefore, differ from teams in other industries as they comprise individuals who are employed by organizations that conduct different businesses. These many separate organizations working together in a close harmony over the duration of the project is fundamental to the overall business of construction. Project success relies heavily on the management of the flow of information among players and access to the right information is vital to efficiently perform the work. Therefore, an efficient information access and effective information exchange methods internally and externally with other organizations needs to be achieved. The model-centric approach introduces a unique solution to solve the problems with the information jungle, inconsistency, and disintegration associated with the traditional document-centric approach. As such, a coherent use of information management is required to merge, comanage, and semantically connect the information and artifacts generated throughout the project phases. Adopting a model-centric approach creates a single point of truth that centralizes information and facilitates access to it. A transformation plan is needed to outline the shift of the construction from document-centric to model-centric. This paper proposes a strategy to support the model-centric transformation of the Architecture, Engineering, and Construction (AEC) industry. Five strategic objectives are outlined and discussed in this paper highlighting considerations that need to be made to enable this transformation. This study describes the "what" necessary to enable and support a model-centric AEC industry.
\end{abstract}

(c) 2020 The Authors. Published by Budapest University of Technology and Economics \& Diamond Congress Ltd Peer-review under responsibility of the Scientific Committee of the Creative Construction Conference 2020.

Keywords: BIM, MBSE, model-centric, system engineering, technology shift

\section{Introduction}

The Architecture, Engineering, and Construction (AEC) industry is one of the most information-intensive industries where information needs to be readily available, accurate, complete, timely, and in a clear format that is understandable by the recipient. The increased complexity of construction projects requires the involvement of a wide range of organizations throughout the lifecycle - from conceptual planning to decommissioning. Project teams, therefore, differ from teams in other industries as they comprise individuals who are employed by organizations that conduct different businesses.

Project success relies heavily on the management of the flow of information among players. The multidisciplinary and fragmented nature of construction creates a challenging environment for successful project implementation. Even with the development of BIM and Systems Engineering, the current practice of construction still relies on the conventional document-centric approach to deliver and manage the data throughout the lifecycle. Joan Allen, a group product manager at Autodesk pointed out that $70 \%$ of construction projects are still using paper as the main medium of communication [1]. This approach 
emphasizes the generation of documents (physical or electronic with restrictive interoperable capabilities) which are exchanged among stakeholders and is prone to inconsistencies and incompleteness. Rezgui and Zarli [2] formulated the following vision statement in relation to transforming the AEC industry: "Construction in the knowledge society uses information and communication technologies as key enablers to meet main societal and industrial challenges. Sustainable construction is driven by total life cycle performance through knowledge intensive and functional integration of products and processes using a model-based approach to pave the way toward a knowledge driven industry." They highlighted that the document-centric information exchange dominating the AEC industry should be replaced with a modelcentric approach [2].

Model-centric approach introduces a unique (and real-world practical) solution to solve the information jungle, inconsistency, and disintegration associated with the document-centric approach. The concept of Model-Based System Engineering (MBSE) was conceived by the International Council on Systems Engineering (INCOSE) to transform engineering by replacing the traditional document-based with a modelbased approach [3]. Adopting a model-centric approach creates a single point of truth that centralizes data $[3,4]$.

Model-centricity provides new opportunities for the development of an integrated platform for information exchanges that can cross the organizational boundaries and provide a unique opportunity for teamwork and efficient information exchange and knowledge-sharing among otherwise isolated entities. While a model-based approach offers flexibility, supports collaboration and communication, automates and centralizes data, it requires changes to the AEC business-as-usual process. Inspired from the five objectives set by the Department of Defense (DoD) in 2018 for the Digital Engineering Strategy [5], this study discusses a strategy to guide the transformation of the AEC industry into a model-based discipline. The five objectives that are discussed in this paper and are as follows: 1) Formalize the development, integration, and use of models; 2) Provide an enduring, authoritative single source of truth; 3) Integrate technological innovation; 4) Establish a supportive infrastructure and environments to collaborate and communicate across stakeholder; and 5) Transform the culture and the workforce to support the transformation. The description of each of the five objectives is supported with previous research endeavours undertaken to explore the transformation of the AEC industry into model-centricity. The objectives are then augmented with a set of considerations to be addressed before achieving model-centricity in the AEC industry.

\section{Overview of System Engineering and Model-Based System Engineering}

Before discussing MBSE and its current applications, it is important to understand what a System and System Engineering is. According to INCOSE, a system "is a construct or collection of different elements that together produce results not obtainable by the elements alone" [6]. Systems can be found in different products across different industries such as automobiles, shuttles, and airplanes. An aircraft, for example, is composed of various systems that are enclosed in a single body. These systems can be mechanical, electrical, and electronics. A F-35 fighter jet, for instance, has 8.5 millions of lines codes and thousands of electric circuit systems [7]. For an airplane to function and fly safely, these systems must thoroughly be designed, assembled and inspected. Additionally, systems must be coherently integrated - an integration that is achieved through System Engineering [8].

System Engineering as defined by INCOSE [6] is "an engineering discipline whose responsibility is creating and executing an interdisciplinary process to ensure that the customer and stakeholder's needs are satisfied in a high quality, trustworthy, cost efficient and schedule compliant manner throughout a system's entire life cycle". According to [9], a System Engineering roadmap can be summarized into the following five steps: 1) Stakeholders are first identified. Stakeholders can be individuals or groups who are participating in the system development; 2) Stakeholders' requirements and concerns are identified through questions of interest; 3) The system requirement, goals, external relationships and boundaries are later identified; 4) Budgets and schedules are established and maintained along with management and maintenance processes. Consequently, a baseline will be set to fulfill the stakeholders and system requirements; 5) User documentations, risk matrices, testing documents are finally created. 
Researchers noted that there are numerous challenges facing System Engineering especially given the complexity of the design of today's systems [7]. System design complexity is increasing faster than it can be controlled, especially as it is assembled from different design parts and not from a common architecture. Moreover knowledge and communication are lost across the project lifecycle, and between different projects, resulting in products that are expensive to test, have low quality, and are risky to operate [10].

The increase of system design complexity forced engineers to reconsider the whole system development phase, and as a result, MBSE emerged. MBSE was first introduced by Wymore and is described as a paradigm that places the model at the center. [3] noted that although MBSE has been extensively researched in the past decade, it remains a mysterious concept that has different meanings to different stakeholders. INCOSE initially defined MBSE as "the formalized application of modeling to support system requirements, design, analysis, verification, and validation activities beginning in the conceptual design phase and continuing throughout the later lifecycle phases" [6]. [4] augmented on the definition put forth by INCOSE and defined MBSE as "the formalized application of modeling (static and dynamic) to support system design and analysis, throughout all phases of the system lifecycle, through the collection of modeling languages, structures, model-based processes, and presentation frameworks used to support the discipline of systems engineering in a model-based or model-driven context".

In MBSE, the "model" is the single source of truth promising increased effectiveness in developing complex systems [11]. A model is a representation of a system that evolves as the lifecycle of the system is progressing. A model can include design information, specifications, and testing requirements all stored in a central location [9]. These models will have different viewpoints, offering a flexibility in viewing model information [12]. For instance, the information viewed by the owner is different from the information viewed by engineer. The owner would be interested in cost information, project risk, on the other hand, the engineer would be interested in material delivery dates, progress, and upcoming weekly tasks. Therefore, model-centric design can capture different system aspects, while offering specific views through different lenses that highlight specific information in the model. In MBSE, models are created using modeling languages. One of the most prevailing languages is Systems Modeling Language (SysML), which is a graphical modeling language developed by Object Modeling Group (OMG) and INCOSE [8]. SysML can be used to analyze, design, and verify complex systems, whether it is software or hardware related.

One of the main reasons driving this shift to MBSE is to solve communication breakdowns that often occur as a result of the traditional document-centric System Engineering approach. In System Engineering, the data is stored in different formats using different software tools, which are deployed across different computers at the workplace. One of the main challenges arise when there are multiple versions of important documents being shared, which causes confusion between different stakeholders and can ultimately lead to conflicts. In addition, important design information is often not properly documented [7]. In a model-centric approach, on the other hand, the information is integrated into the model which is connected to the different requirements and design databases. The model can be then used to assess the performance of the system [13]. If changes in the requirements or design occur, it can be checked whether these modifications are safe to be implemented. If they are approved, they will be automatically updated into the main model. Therefore, the capabilities offered by MBSE help prevent design errors, by enabling a proactive check and analysis of the requirements and improving design feedback time [14]. MBSE is envisioned to transform a document-centric discipline into a model-centric discipline where data is maintained within a single repository and each stakeholder has a singular definition, with all needed attributes and relationships stakeholders are outlined [3].

\section{A Model-Centric Transformation Strategy for the AEC industry}

Trying to solve the challenges of complex systems, other industries adopted the application of MBSE [9]. The AEC industry is facing similar challenges while still operating in a document-centric manner. Even the great effort in researching multidimensional BIM in the last decades didn' $t$ lead to a sufficient improvement in information flow [15]. The chance towards a model-centric approach becomes a necessity for the AEC industry to solve the long existing problems. To apply MBSE to the requirements of the construction industry a customized transformation strategy is needed, taking in consideration that there are currently 
no existing implementation frameworks. This paper discusses five objectives adapted from the Digital Engineering Strategy set by the DoD [5].

\subsection{Objective \#1 - Formalize the development, integration, and use of models}

As mentioned in Section 2, a model "represents reality for the given purpose" [16]. Following the first objective of this study, the context of models in construction needs to be developed to create a common understanding and to evolve into a model-centric industry.

As models are a representation of something, they only have properties to the given purpose. Therefore, the purpose of the model and the relevant stakeholders must be considered [17]. Modeling construction information does not strictly refer to Computer-Aided-Drawings (CAD), Building Information Modeling (BIM), and reality models, but rather it references the representation of construction process (from conceptual planning to demolition) in data to facilitate exchange and interoperability of project information. Models could be 3D models, 4D simulations, drawings, specifications, Request for Information's (RFIs), cost, schedule, progress reports, as-builts [1]. A construction information model also consists of different repositories, which can then be accessible to different stakeholders [17]. Each model is tailored for its intended purpose and stakeholders and serves to understand the system, to optimize the behavior if necessary and / or design strategies for future operations [18]. Additionally, simulated models are used either because the real system is not yet defined or available or because it cannot be executed directly due to cost, time, resource or risk restrictions. These simulations, for example, enable design analysis on models and allow what-if analysis to be carried out [19].

It should be noted that while evolving technology enables more complex and capable models, it may not improve effectiveness if the human factor is not appropriately considered [2]. Humans have cognitive and conceptual limitations that limit the amount and type of information they can effectively comprehend and use to make decisions. Designing for humans requires understanding their capabilities and limitations and identifying their information needs so that the model intelligence can extend the overall system intelligence $[16,20]$.

\subsection{Objective \#2 - Provide an enduring, authoritative single source of truth}

Research efforts conducted during the past decades show that while modeling has already moved beyond the $\mathrm{n}$-dimensional development of BIM, the degree of integration between different systems and stakeholders can still be greatly improved [15]. Given the various use-cases applied throughout the construction project lifecycle and their diverse requirements, there is currently little interoperability and automation potential to leverage the use of BIM [15]. Although the advances in vendor-independent formats such as Industry Foundation Classes (IFC) have a significant impact on the development of integrated methods for the exchange of construction data [15]. IFC provide the standards for model-based interoperability, similar to how SysML is becoming the standard modeling language in MBSE [25]. While taking into consideration industry's stakeholders, subsets of the IFC scheme - so-called Model View Definitions (MVDs) - are created to meet the requirements of various disciplines [30]. Although these schemes are not yet fully developed, together with Information Delivery Manuals (IDMs), they support collaborative project management [30]. Research efforts show for example how Building Automation System (BAS) based systems which are used in facility management can be represented in an IFC structure [21]. While other researchers are still sceptical and consider that even with the further development of IFC, it does not seem feasible to switch from static BIM data to a dynamic web-based system [15]. Therefore, there are other approaches based on linking data which is kept in its original format [22].

Examining developments in other industries has led construction researchers to focus efforts towards a more holistic approach across the lifecycle of a construction project - the concept of Digital Twin. As the authors have shown in [23], the concept of the Digital Twin in the AEC industry goes beyond BIM. The authors classify the model-related body of knowledge in construction into three subcategories: Digital Models (most notably illustrated through BIM where there is no automated links between physical object and virtual representation is to be counted), Digital Shadow, and Digital Twin (highest level of data integration). Digital Twins in their full form have an automated bidirectional link between the physical and 
digital object [23]. The use of BIM in construction projects nowadays can thus be the starting point for the progression of sustainable Digital Twins that are enriched by data collected throughout the project lifecycle [15]. Regardless of the current maturity level of the Digital Twin concept in construction, the exchange of information continues to develop into more open, web-based platforms through the use of open formats such as IFC but also enabled by linking existing data. This direct access to information increases the importance of a single source of truth, which MBSE will offer if implemented.

[24] defined a single source of truth as an authoritative source of data that offers data services to other entities. In construction, a single source of truth is thought of as a Common Data Environment (CDE) and the practice of properly storing data such as plans, correspondences, specifications, Computer-Aided Drawings (CAD), 4D simulations in one place without duplications and with a clear path to locate, extract, and share the data throughout the lifecycle of the project - from design, to planning, construction and operations [1,25]. A single source of truth can be leveraged not only by the dynamic data that a Digital Twin manages, but also by the constant knowledge accumulated about the physical objects, which can lead to new insights and patterns with the use of data analysis $[15,26]$.

[27] noted that a shared common data environment (CDE) or data repository is required to reduce miscommunication. Even though platforms that meet these requirements are less common in construction than in other industries, the use of CDEs is growing. Bentley offers such a platform with ProjectWise and Autodesk with BIM 360. Both of these platforms are constantly evolving, and their features, interoperability in particular, are being upgraded and tailored to meet the requirements of the AEC industry and its stakeholders $[1,28]$. With the ongoing development towards using a single source of truth in construction projects new challenges arise. Whyte pointed out that simulations based on wrong assumptions can lead to disasters, and thus, it must be ensured that the single source of truth is reliable and that verification and validation processes are put-in place and carried out throughout the lifecycle of the construction project [26].

\subsection{Objective \#3 - Integrate technological innovation}

The construction industry is not an exception to the pervasive digital revolution. The industry is dealing with large volumes of data arising from different disciplines throughout the lifecycle of a capital project. As the Internet continues to evolve, and with the increased growth in the number of connected devices - coined as IoT, there is a massive increase in the accompanied data traffic volume. The vast accumulation of construction data, including BIM data and IoT data has pushed construction to enter the era of Big Data [29]. Big Data has three attributes; namely volume, variety, and velocity (a.k.a. 3V's) [30]. Construction data is typically large, heterogeneous (represented in various formats), and dynamic (resulting from the nature of data sources) [31]. The application of the major technologies of Industry 4.0 to a construction project generates multiple scenarios to be considered under the model-centric approach. For example, workers' wearables in construction linked to loT allows the collection of ergonomic data. The gather raw data is stored in a database where data analytics is employed to infer information. This information is then accessed by the responsible person (decision-maker) to make decisions related to the behavior of workers. This decision is then communicated to the workers in the field, whose behavior will be adjusted accordingly, forming a closed loop under the model-centric approach. Visualization technologies such as Virtual Reality and AR and as well as web-based applications can also support the model-centric by leveraging the accessibility to, usage of, and interaction with information.

\subsection{Objective \#4 - Establish a supportive infrastructure and environments to collaborate and communicate across stakeholders}

As outlined under the second objective, the collaboration between the various AEC stakeholders is centered on the idea of a single source of truth that is implemented on a web-based platform. Although the concept of the single source of truth is vital to model-centricity, the question pertaining to how the integration of the numerous stakeholders with their subsystems and their highly specialized applications in this single source of truth can be facilitated is subject of current research. The key to generate a reliable single source of truth is interoperability, which can be achieved by using open formats and also by linking existing data. 
It should be noted that, even when interoperability is achieved within the single source of truth, there are other points to consider, such as the Information Technology (IT) infrastructure and the corresponding hardware and software tools needed to operate the web-based platform. This transformation should be also supported by a team of well-trained individuals and specialists to provide on-going feedback and assistance.

\subsection{Objective \#5 - Transform the culture and train the workforce to support the transformation.}

Research indicates that the level of cultural change needed to transform an industry through MBSE should not be underestimated [32]. This transformation goes beyond the technical aspects of MBSE as it requires strong leadership involvement and commitment. Additionally, stakeholders need to be educated on modelcentricity.

To overcome cultural challenges associated with the shift to a new system, Thales group which is a multinational aerospace company, has applied a rigorous plan related to MBSE implementation [33]. Owner and key players are involved early in the project to define and modify their needs. Then, research and training experts highlight operational needs and produce prototypes that are later validated. Moreover, a dedicated development team is responsible for enhancing the current modelling software. Additionally, dedicated support teams are put in charge to perform most of the analysis that require special attention. MBSE champions are also delegated to help operational engineers implement MBSE. Furthermore, local support teams are assembled and act as the liaison between operational users and support teams. Finally, operational engineers will oversee the implementation plan, and will regularly check and ensure that the current MBSE solutions are helping the engineers [32]. A similar process can be established for the AEC industry and tailored to the specifics of the construction project.

\section{Considerations to support the Model-Centric Transformation for the AEC industry}

The shift towards a model-centric industry is a necessity to continue to exist in the growing market, however the current state of the AEC industry is still mainly document-centric. The previous section discussed five objectives for implementing a model-centric approach. This section outlines several considerations that need to be addressed and should be carefully thought of when discussing model-centricity. These considerations can be summarized into the five themes illustrated in Fig. 1 (central figure) and are discussed in detail in the following sections.

\subsection{Objective \#1 - Formalize the development, integration, and use of models}

As different stakeholders are involved throughout the lifecycle of a construction project, it is important to study and explore their relationships among each other. Their needs and requirements for the models should be well considered, defined and formalized. It is also crucial to define a common lexicon for models in the AEC industry to ensure consistency and buy-in from all stakeholders. After defining and agreeing on what the models are, the realization of those models on construction projects should be formalized. This ensures that the modes are complete, trustworthy, accurate and meet the specified requirements of the stakeholders. These models will be used to communicate and collaborate throughout the project. Another important aspect that should be considered is the Project Delivery System. Given the complex and dynamic nature of the AEC industry, it becomes critical to explore the feasibility of adopting a model-centric approach with the different Project Delivery Systems. While the adoption of a model-centric approach can be aligned with the Integrated Project Deliver System where communication, collaboration, and transparency are supported contractually, a significant number of construction projects are executed under other delivery systems such as Design-Build and Construction Management. Additionally, as the AEC industry prepares to become model-centric, models become valuable assets throughout the project lifecycle, requiring management and control (model curation). 


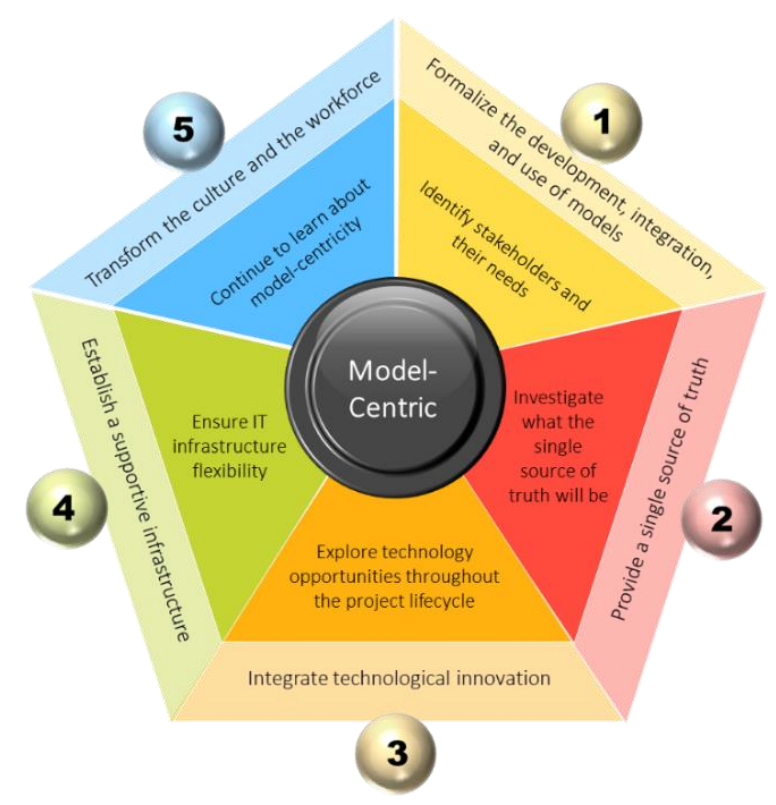

Fig.1. The AEC model-centric Tranformation Objectives and Considerations (objectives are listed in outer pentagon and considerations are listed in the inner pentagon )

\subsection{Objective \#2 - Provide an enduring, authoritative single source of truth}

After identifying the requirements of the different stakeholders, the single source of truth that will serve as the central reference point throughout the project lifecycle needs to be defined. With the existence of various types of models and software, the integration of models in a single source of truth is the main challenge. Main research efforts are undertaken to connect and link these models to apply the modelcentric approach and establish a master model that will act as a hub for all other models.

To ensure the proper use of the single source of truth as the project moves forward, a governance plan must be established to trace and capture changes. It should be also noted that having a central model does not mean that everyone and anyone has access to the same information. Different viewpoints are crucial in order the channel the proper information to the right stakeholders.

\subsection{Objective \#3 - Integrate technological innovation}

Shifting towards Construction 4.0 requires the adoption of MBSE into the AEC industry. However, the transformation is not linear as it depends on the use of advanced technology such as AR, IOT and Big Data. Technological advances are rapidly changing, and thus, it is important to make wise investments. When making technology-related decisions, it is important to ensure that knowledge is continuously captured, used, and leveraged, to provide the industry with a competitive edge to adapt and grow.

\subsection{Objective \#4 - Establish a supportive infrastructure and environments to collaborate and communicate across stakeholders}

In order to integrate advanced technologies into a project or a process, a robust IT infrastructure must exist. Investing in technology and infrastructure is as important as investing in other decisions. Thus, the IT infrastructure can be thought of as the main "mechanism" to connect stakeholders and exchange information. Not having a robust and secure IT infrastructure will overturn all the advances in the construction lifecycle. Investing in establishing communication channels between stakeholder will help promote model-centricity by punching holes in the wall of silos of the AEC industry. Having subject matter experts guide and assist the development of an IT infrastructure and having leadership invest and support this initiative is crucial to this objective.

\subsection{Objective \#5 - Transform the culture and train the workforce to support the transformation}

The human factor is as important as the use of technology. In order to exploit the full potential of technology not only the leadership level needs to be brought on board, but the workforce needs to be trained to 
navigate model-centricity. As this transformation requires collaboration and transparency among the stakeholders, it is important to ensure buy-in and support from all project participants. Project team leaders and model-centricity champions can also develop guidelines and organize workshop to educate and train stakeholders. Other industries, particularly manufacturing, have always been a source of innovation for the $A E C$ industry. AEC professionals can learn from other industries that have benefited from a model-centric transformation and adopt and adjust best practices to fit the industry. Moreover, companies must realize that such a transformation is a long-term goal and efforts and investment in all aspects need to happen continuously and incrementally, over a long period of time. Such a transformation is not instantaneous, and although the cost of change is high, the return of investment will be higher.

\section{Conclusions and Future Work}

The increased complexity of construction projects along with the increased customer expectation has added more pressure on the AEC industry to re-examine its practices and revolutionize and modernize how it plans, designs, executes, and maintains its projects. The multi-party nature and uniqueness of the architecture, engineering, and construction industry creates a challenging environment for successful project implementation. Project success relies heavily on timely transfer of the correct information among players. Model-centricity provides new opportunities for the development of an integrated platform for information exchanges that can cross the organizational boundaries and provide a unique opportunity for teamwork and workflow automation among otherwise isolated entities. Characteristics of this new modelcentric approach are centered on the concepts of ownership, accessibility, availability, and timeliness of information together with the notions of process digitization, integration, and optimization. This paper discussed five objectives that are envisioned to enable the transformation of the AEC industry to a modelcentric industry, namely: 1) Formalize the development, integration, and use of models; 2) Provide an enduring, authoritative single source of truth; 3) Integrate technological innovation; 4) Establish a supportive infrastructure and environments to collaborate and communicate across stakeholder; and 5) Transform the culture and the workforce to support the transformation. On the other hand, these objectives cannot be achieved, without trying to mitigate the five identified constrains. First the stakeholders and their needs must be established, second a central model must be generated which provides different viewpoints to the different stakeholders. Advanced technology can be implemented to create and exchange knowledge and analyse data. Collaboration and integration will lead to change the silo culture in the AEC industry. Finally, the human aspect and training needs and support must be emphasized to achieve lasting change. While this research investigates the feasibility of a model-centric transformation in the AEC industry from a broad perspective, future research could elaborate on the objectives and considerations presented in this paper and provide a more focused and technical assessment of such transformation.

\section{References}

[1] Allen, J., 2019. A Single Source of Truth: BIM 360 Document Management.URL https://www.autodesk.com/autodeskuniversity/class/Single-Source-Truth-BIM-360-Document-Management-2019-0\#video (accessed April 14, 2020).

[2] Rezgui, Y. and Zarli, A., 2006. Paving the Way to the Vision of Digital Construction: A Strategic Roadmap. Journal of Construction Engineering and Management, 132(7), pp.767-776. Available at: https://doi.org/10.1061/(asce)0733-9364(2006)132:7(767).

[3] Vaneman, W.K., 2018. Evolving Model-Based Systems Engineering Ontologies and Structures. INCOSE International Symposium, 28(1), pp.1027-1036. Available at: https://doi.org/10.1002/j.2334-5837.2018.00531.x.

[4] Vaneman, W.K., 2016. Enhancing model-based systems engineering with the Lifecycle Modeling Language. 2016 Annual IEEE Systems Conference (SysCon). Available at: https://doi.org/10.1109/syscon.2016.7490581.

[5] Deputy Assistant Secretary of Defense Systems Engineering, 2018. Department of Defense Digital Engineering Strategy.).

[6] INCOSE, 2007. Systems Engineering Vision 2020.

[7] D'Ambrosio, J. and Soremekun, G., 2017. Systems engineering challenges and MBSE opportunities for automotive system design. 2017 IEEE International Conference on Systems, Man, and Cybernetics (SMC). Available at: https://doi.org/10.1109/smc.2017.8122925.

[8] Hallqvist, J. and Larsson, J., 2016. Introducing MBSE By using Systems Engineering Principles. INCOSE International Symposium, 26(1), pp.512-525. Available at: https://doi.org/10.1002/j.2334-5837.2016.00175.x.

[9] Madni, A.M. and Sievers, M., 2017. Model-Based Systems Engineering: Motivation, Current Status, and Needed Advances. Disciplinary Convergence in Systems Engineering Research, pp.311-325. Available at: https://doi.org/10.1007/978-3-319-62217-0_22..

[10] Bayer, T., 2017. Progress check: how well is the planned Europa's Mission's MBSE application addressing systems engineering challenges?. URL http://hdl.handle.net/2014/47456 (accessed 4.14.20). 
[11] Tepper, N.A., 2010. Exploring the use of Model-based Systems Engineering (MBSE) to develop systems architectures in naval ship design (Doctoral dissertation). Master's Thesis, Dept. Mech. Eng., MIT, Cambridge, MA, USA..URL http://hdl.handle.net/1721.1/61910 (accessed 4.14.20).

[12] Chami, M., Aleksandraviciene, A., Morkevicius, A., and Bruel, J.-M., 2018. Towards Solving MBSE Adoption Challenges: The D3 MBSE Adoption Toolbox. INCOSE International Symposium, 28(1), pp.1463-1477. Available at: https://doi.org/10.1002/j.2334-5837.2018.00561.x.

[13] Friendenthal, S., Steiner, R., and Moore, A., 2009. A Practical Guide to SysML: The Systems Modeling Language. Kybernetes, 38(1/2). Available at: https://doi.org/10.1108/k.2009.06738aae.004.

[14] Zimmerman, P., 2014. A Review of Model-Based Systems Engineering Practices and Recommendations for Future Directions in the Department of Defense, in: 2nd Systems Engineering in the Washington Metropolitan Area (SEDC 2014) Conference, Chantilly, VA.

[15] Boje, C., Guerriero, A., Kubicki, S., and Rezgui, Y., 2020. Towards a semantic Construction Digital Twin: Directions for future research. Automation in Construction, 114, p.103179. Available at: https://doi.org/10.1016/j.autcon.2020.103179

[16] Blackburn, M., Bone, M. and Witus, G., 2015. Transforming System Engineering through Model-Centric Engineering. Available at: https://doi.org/10.21236/ada631778.

[17] Weilkiens, T., 2013. What is a model?. https://mbse4u.com/2013/03/15/what-is-a-model/.(accessed 4.14.20).

[18] Dickopf, T., Apostolov, H., Müller, P., Göbel, J.C., Forte, S., 2019. A Holistic System Lifecycle Engineering Approach - Closing the Loop between System Architecture and Digital Twins. Procedia CIRP, 84, pp.538-544. Available at: https://doi.org/10.1016/j.procir.2019.04.257.

[19] Zilvinas, S., and Saulius, P., 2017. Building Executable SysML Model - Automatic Transmission System (Part 1) [WWW Document]. URL https://blog.nomagic.com/building-executable-sysml-model-automatic-transmission-system-part-1/ (accessed 4.15.20).

[20] German, E.S., 2017. An Investigation of Human-Model Interaction for Model-Centric Decision-Making 132

[21] Tang, S., Shelden, D. R., Eastman, C. M., Pishdad-Bozorgi, P., and Gao, X., 2020. BIM assisted Building Automation System information exchange using BACnet and IFC. Automation in Construction, 110, p.103049. Available at: https://doi.org/10.1016/j.autcon.2019.103049.

[22] Hu, S., Corry, E., Curry, E., Turner, W.J.N., and O'Donnell, J., 2016. Building performance optimisation: A hybrid architecture for the integration of contextual information and time-series data. Automation in Construction, 70, pp.51-61. Available at: https://doi.org/10.1016/j.autcon.2016.05.018.

[23] El Jazzar, M., Piskernik, M., and Nassereddine, H. ,2020. Digital Twin in construction: An Empirical Analysis

[24] Pang, C. and Szafron, D., 2014. Single Source of Truth (SSOT) for Service Oriented Architecture (SOA). Lecture Notes in Computer Science, pp.575-589. Available at: https://doi.org/10.1007/978-3-662-45391-9_50.

[25] Ellis, G.,2019. SSOT: Why Everyone in Construction Should Care About this Acronym, PlanGrid Constr. Product. Blog.URL https://blog.plangrid.com/2019/07/single-source-of-truth-in-construction/ (accessed April 14, 2020).

[26] Whyte, J., 2016. The future of systems integration within civil infrastructure: A review and directions for research. INCOSE International Symposium, 26(1), pp.1541-1555. Available at: https://doi.org/10.1002/j.2334-5837.2016.00244.x.

[27] hen, Y., Jupp, J., 2019. BIM and Through-Life Information Management: A Systems Engineering Perspective, in: Mutis, I., Hartmann, T. (Eds.), Advances in Informatics and Computing in Civil and Construction Engineering. Springer International Publishing, Cham, pp. 137-146. Available at :https://doi.org/10.1007/978-3-030-00220-6_17

[28] Bentley Systems, Saulius, P., 2020. ProjectWise Lead Your Firm Into the Era of Digital Project Delivery [WWW Document]. URL https://www.bentley.com/en/products/brands/projectwise (accessed 4.15.20).

[29] Williams, N., P. Ferdinand, N., and Croft, R., 2014. Project management maturity in the age of big data. Int. J. Manag. Proj. Bus. 7, 311-317. Available at: https://doi.org/10.1108/IJMPB-01-2014-0001

[30] Bilal, M., Oyedele, L. O., Qadir, J., Munir, K., Ajayi, S. O., Akinade, O. O., Owolabi, H.A., Alaka,H. A., Pasha, M., 2016. Big Data in the construction industry: A review of present status, opportunities, and future trends. Advanced Engineering Informatics, 30(3), pp.500-521. Available at: https://doi.org/10.1016/j.aei.2016.07.001.

[31] Aouad, G., Kagioglou, M., Cooper, R., Hinks, J., and Sexton, M., 1999. Technology management of IT in construction: a driver or an enabler? Logistics Information Management, 12(1/2), pp.130-137. Available at: https://doi.org/10.1108/09576059910256583.

[32] Bonnet, S., Voirin, J.-L., Normand, V., and Exertier, D., 2015. Implementing the MBSE Cultural Change: Organization, Coaching and Lessons Learned. INCOSE International Symposium, 25(1), pp.508-523. Available at: https://doi.org/10.1002/j.23345837.2015.00078.x.

[33] Redmond, A.M., 2018. Measuring the Performance Characteristics of MBSE Techniques with BIM for the Construction Industry. 2018 11th International Conference on Developments in eSystems Engineering (DeSE). Available at: https://doi.org/10.1109/dese.2018.00047. 